\title{
Effects of weather and landscape on the equine West Nile virus infection risk in Mississippi, USA
}

\author{
Guiming Wang \\ Department of Wildlife, Fisheries and Aquaculture, Mississippi State University, Starkville, \\ MS, USA
}

\begin{abstract}
The West Nile virus (WNv) continues to be a public health concern in North America. Dry weather appears to increase human WNv infection risks, but it is uncertain whether dry weather conditions exert similar effects on the corresponding equine WNv infection. This study assessed the effects of precipitation of the previous year and land cover diversity on the equine WNv risk of Mississippi, USA, at the county level in the year 2002 using Bayesian hierarchical models. The risk estimated for 2002 was found to be inversely related to annual precipitation of the preceding year. Equine WNv risks were lower with greater land cover diversity probably due to the diluting effects of biodiversity. Correlation between the equine and human WNv risks was positive but relatively low. Dry weather conditions of the previous year might reduce mosquito competitors and predators and subsequently increase mosquito abundances and equine WNv risks in agricultural areas with low biodiversity.
\end{abstract}

Correspondence: Guiming Wang, Department of Wildlife, Fisheries and Aquaculture, Mail Stop 9690, Mississippi State University, Starkville, MS 39762, USA

Tel: +01.662.325.0414 - Fax: +01.662.325.8276.

E-mail: guiming.wang@msstate.edu

Key words: Bayesian hierarchical models; Integrated nested Laplace approximations; Land cover diversity; Spatial models; Dilution effect hypothesis.

Acknowledgments: the author is grateful to Dr. Charles Wax for providing climate data. Anonymous reviewers made helpful, constructive comments on this manuscript.

Funding: the author was supported by Department of Wildlife, Fisheries and Aquaculture and the Forest and Wildlife Research Center, Mississippi State University.

Conflict of interest: the author declares no potential conflict of interest.

Received for publication: 20 March 2015.

Revision received: 29 June 2015.

Accepted for publication: 10 August 2015.

(C) Copyright G. Wang , 2015

Licensee PAGEPress, Italy

Geospatial Health 2015; 10:357

doi:10.4081/gh.2015.357

This article is distributed under the terms of the Creative Commons Attribution Noncommercial License (by-nc 3.0) which permits any noncommercial use, distribution, and reproduction in any medium, provided the orig-

\section{Introduction}

The invasion of West Nile virus (WNv) in the USA has exemplified the rapid spread of invasive, infectious diseases in non-native ranges. The first human WNv infection in the USA was reported in New York City in 1999 (Nash et al., 2001). Rapidly, 14,539 equine cases of WNv infections were reported in 40 states including the District of Columbia during 2002 (0'Leary et al., 2004). Human WNv outbreaks occurred in USA again in 2012 (DeGroote et al., 2014) and the disease remains as a public health concern (Harrigan et al., 2014).

West Nile virus infection threatens health and survival of humans and domesticated mammals, causing neuroinvasive diseases in mammalian hosts (0'Leary et al., 2004; Ward and Scheurmann, 2008). The fatality rate of equine WNv infection can be as high as 30\% (Schuler et $a l ., 2004)$. The virus is mainly transmitted in a bird-mosquito enzootic cycle with birds as amplifying hosts and horses and other mammals as dead-end hosts. Mammals are infected when bitten by WNv-infected mosquitoes (Hayes et al., 2005; LaDeau et al., 2008; Ward and Scheurmann, 2008). Aedes aegypti, A. albopictus, Culex pipiens, $C$. quinquefasciatus, Ochlerotatus socllicitans, 0 . triseriatus, and Psorophora columbiae are the main insect vectors responsible for the transmission of WNv from birds to mammals in Mississippi, particularly $C$. pipiens and $C$. quinquefasciatus (Cooke et al., 2006; Andreadis, 2012). The abundance of birds and mosquitoes may affect the transmission rate of WNv from infected birds to mammalian hosts assuming more interaction between infected and susceptible individuals at greater densities. Therefore, environmental factors influencing the abundance of WNv hosts and vectors may affect WNv transmission rates in birds and mammals (Epstein and Defilippo, 2001; Mongoh et al., 2007). Chase and Knight (2003) have hypothesized that droughts of the previous year would increase the abundance of mosquitoes by reducing the predators and (or) competitors of the mosquitoes. Low spring precipitation is associated with high WNv seroprevalence in horses in Israel (Aharonson-Raz et al., 2014). Wang et al. (2010) found that dry weather conditions of the previous year seem to increase human WNv infection risks in Mississippi in 2002, while Walsh (2012) found a negative impact of precipitation on the rate of human WNv diseases in New York State, USA. However, it is unknown whether prior year's precipitation is indeed a predictor of equine WNv infections.

Habitat conditions may also affect the abundance and species composition of WNv's avian hosts. For example, high plant diversity or patch heterogeneity may increase avian species richness (MacArthur, 1964). The dilution effect hypothesis posits that increases in species diversity of disease hosts would dilute the host effect of pathogens and reduce the infection prevalence of disease in vector and host populations (Ostfeld and Keesing, 2000). LoGiudice et al. (2003) found that more diverse tick (Ixodes scapularis) host communities had more host species that were less competent reservoirs of Lyme disease. Those less competent reservoirs dilute the effects of the rodent Peromyscus leucopus, the most competent reservoir, and thus reduce the vector 
infection prevalence of Lyme disease. Furthermore, Ezenwa et al. (2007) found that greater shrub land areas and less wetland areas enhance avian diversity and the number of nonpasserine birds (i.e., non-competent hosts) and thus reduce WNv infection prevalence rates of Culex mosquitos in Louisiana, USA. Therefore, it is plausible to predict that land cover diversity would increase the species diversity of avian communities and thus reduce equine WNv infection risks (Roche et al., 2012). Equine WNv infections varied spatially at the county level in the American states of North Dakota, Texas and Florida (Mongoh et al., 2007; Ward and Scheurmann, 2008; Rios et al., 2009). Equine and human WNv vectors may differ in abundance and may be differently influenced by weather conditions. However, little is known about the spatial correlation between the WNv infection risks of humans and horses with the respective spatial auto-correlation of human and equine WNv infections being accounted for. The objectives of this study were to: i) estimate the relative risk of equine WNv in Mississippi at the county level using Bayesian hierarchical spatial models with conditional auto-correlative (CAR) distributions to account for spatial autocorrelation; ii) evaluate the effects of prior year's precipitation and land cover diversity on the relative risk of equine WNv infection; and iii) test for spatial correlation of WNv infection between humans and equines using multivariate CAR distributions. Although data were collected 10 years ago, the findings of this study on the initial outbreaks of equine WNv infections in USA may provide insight into the effects of environmental conditions on the initial outbreaks of invasive zoonotic diseases in the future.

\section{Materials and Methods}

With the aim to understand the effects of weather and landscape diversity on the initial outbreaks of invasive zoonotic diseases, we chose the 2002 data on human and equine WNv infections, because the 2002 census of horses provided the exact numbers of horses in each county facilitating the estimation of the number of WNv-infected horses using Poisson distribution. The equine census is conducted only every five years. Additionally, the national land cover classification in USA is updated about every 10 years. The 2001 National Land Cover Database (NLCD) was based on the 2000 Landsat imagery (http://www.mrlc.gov/nlcdrlc.php). The lack of frequent equine census data and frequent land cover data limited the use of equine WNv infection data for other years.

The number of horses for 80 of the 82 counties in Mississippi for 2002 were downloaded from the 2002 Census of Agriculture of the United State Department of Agriculture National Agricultural Statistic Services website (http://www.agcensus.usda.gov) together with the numbers of equine WNv cases of all 82 counties, downloaded from the Mississippi State Department of Health data archive website (http://www.msdh.state.ms.us/msdhsite/_static/resources/480.pdf). Total annual precipitation for each county in 2001 was used to assess delayed effects of precipitation on the equine $\mathrm{WNv}$ infection risk. Annual precipitation data were provided by state climatologists for 73 Mississippi counties (Wang et al., 2010). Annual precipitation of nine counties with missing precipitation records during 2001 was interpolated with an ordinary Kriging method (Ribeiro and Diggle, 2001; Wang et al., 2010).

The 2001 NLCD was used to compute the Shannon-Weiner diversity index for each of the 82 counties in Mississippi (www.mrlc.gov/nlcd2001.php). Mississippi has 15 land cover types according to the 2001 NLCD: water; developed area I-IV; deciduous for- est; evergreen (or pine) forest; mixed forest; shrub, grassland; pasture; crop; woody wetland; and emergent herbaceous wetland (Homer et al., 2007). Proportions of land cover types were first computed using the geographic information system IDRISI Taiga, version 15.0 (Clark Labs, Clark University, Worcester, MA, USA). Then Shannon-Weiner diversity index was calculated with the formula:

$$
d i v=-\sum_{i=1}^{15} p_{i} \ln p_{i}
$$

for each county, where $p_{i}$ is the proportion of land cover type i in a county; and $\ln p_{i}$ the natural logarithm of $p_{i}$.

With the assumption that the observed number of equine WNv cases follows a Poisson distribution, the mean number of equine WNv cases reported during 2002 can be modelled as a product of the expected rate and relative risk in a county as follows:

$$
O_{i} \sim \text { pois }\left(\lambda_{\mathrm{i}}\right) \text { and } \lambda_{\mathrm{i}}=e_{i} \theta_{i}
$$

where $\lambda_{i}$ is the Poisson parameter of the $i$ th county; $e_{i}$ the expected rate of the $i$ th county and $\theta_{i}$ the relative risk. The expected rate $e_{i}$ was calculated as a product of the total number of horses of a county and statewide rate of equine $\mathrm{WNv}$ diseases in 2002 . The relative risk, $\theta_{i}$, was modelled as a function of environmental variables, e.g., annual precipitation of the previous year and Shannon-Weiner diversity index of land cover.

The expected numbers of equine WNv cases served as reference risk levels, based on the state-level average, for estimating the local or county-specific relative risks. A county was considered to have equine WNv risk greater than expected if the estimate of $\theta$ was greater than 1 . Excess zeros of WNv infection cases result in extra variability in observations, violating the assumption of Poisson distributions that mean is equal to variance. Therefore, the zero-inflated Possion (ZIP) models with log link function were used to account for excess zero cases and unreported cases.

The null (eq. 3) and full (eq. 4) models of equine WNv risk were of the form:

$$
\begin{aligned}
& \log _{e} \lambda_{i}=\log _{e} e_{i}+\alpha+v_{i+} u_{i} \\
& \log _{e} \lambda_{i}=\log _{e} e_{i}+\alpha+\beta_{1} \operatorname{prec}_{i}+\beta_{2} \operatorname{div}_{i}+v_{i+} u_{i}
\end{aligned}
$$

where $\alpha$ is an intercept; $\beta_{l}$ the regression coefficient of annual precipitation of previous year $\left(\right.$ prec $\left._{i}\right) ; \beta_{2}$ coefficient for land cover diversity index $\left(\operatorname{div}_{i}\right) ; v_{i}$ a normally distributed, spatially independent random variable, $v_{i} \sim N\left(0, \sigma^{2}{ }_{v}\right)$, representing spatially uncorrelated heterogeneity (UH); and $u_{i}$ a spatially structured random variable representing the effects of the first-order neighboring counties [i.e., the spatially correlated heterogeneity $(\mathrm{CH})]$ on the relative risk $(\theta)$.

Extra variability, which often is observed in the number of infection cases of an infectious disease, was accounted for by the unstructured and structured heterogeneity $v_{i}$ and $u_{i}$ in equations 3 and 4 . Conditionally auto-correlative distributions were used to model the $\mathrm{CH}$ effect in the relative risk (Lawson et al., 2003). In the CAR model, estimates of relative risk are not necessarily positively related to numbers of cases because predicted numbers of cases are the smoothing estimates over those of the first order neighboring counties (Lawson et al., 2003).

The unknown parameters and the $\mathrm{UH}$ and $\mathrm{CH}$ effects of the candidate models were estimated using a combination of the Besag spatial 
effect model (CH) and independent random variable model (UH) within the R package INLA (Rue et al., 2009). The priors of variances for the $\mathrm{CH}$ and $\mathrm{UH}$ components were log gamma distributions (Blangiardo et al., 2013), such as:

$$
\begin{aligned}
& \log \left(1 / \sigma_{v}^{2}\right) \sim \log \text { gamma }(1,0.0001), \\
& \log \left(1 / \sigma_{u}^{2}\right) \sim \log \text { gamma }(1,0.0001) .
\end{aligned}
$$

Four candidate models were built by adding variables to the ZIP null model (eq. 3) in a forward stepwise manner to assess the effects of precipitation and land cover diversity on equine WNv risk. The deviance information criterion (DIC) was used to select the best approximate models among the four candidate models (Spiegelhalter et al., 2002a). The DIC weight, an equivalent to Akaike weight, and $\triangle \mathrm{DIC}$ were also calculated for each model (Burnham and Andreson, 2002). The $\Delta \mathrm{DIC}$ of a model is the difference between the DIC value of the model and the lowest DIC value among all candidate models. The best approximate model has the lowest DIC value or highest DIC weight, and the $\triangle \mathrm{DIC}$ of a competing model is at $<2$.

Multivariate conditional auto-correlative (MCAR) models (eq. 5) were applied to estimate the spatial correlation between equine and human WNv infection risks (Lawson, 2009):

$$
\log \left(\lambda_{i}[k, 1]\right)=\log \left(\mathrm{e}_{i}[k, 1]\right)+\alpha_{i}[k, 1]+\mathrm{W}_{i}[k, 1]+\mathrm{U}_{i}[k, 1]
$$

where subscript $i$ and index $k$ are for the $i$ th county and the $k$ th disease, respectively. The letters $\lambda, \alpha$, and $e$ denote the Poisson mean, intercept, and expected counts of a disease, respectively, as in the univariate model (eq. 3); $U_{i}$ is a vertical vector of random variables of multivariate normal distributions for the $i$ th county, with a mean vector of zeros and covariance matrix $\Sigma_{2 \times 2}, U_{i} \sim M N V\left(0_{2 \times 1}, \Sigma_{2 \times 2}\right)$; and $W_{i}$ is a vertical vector of random variables of multivariate CAR distributions for the $i$ th county, i.e., $W_{i} \sim \operatorname{MCAR}\left(1_{2 \times 1}, \Omega_{2 \times 2}\right)$. Bivariate random variables $U$ and $W$ represent the spatially unstructured and structured heterogeneity of relative risks, respectively, like their univariate counterparts $u$ and $v$ in eq.3. Correlation between relative equine and human WNv risks (CR) was estimated as:

$$
C R=\frac{R_{1}[] \cdot R_{2}[] / N-\bar{R}_{1} \bar{R}_{2}}{s_{R_{1}} s_{R_{2}}}
$$

where the symbol $R_{I}[]$ is a vector of relative risks of equine WNv for 80 counties; $R_{2}$ [ ] a vector of relative risks of human WNv; $\bar{R}_{1}$ and $\bar{R}_{2}$ the means of the relative risks of equine and human WNv over 80 counties; $S_{R_{1}}$ and $S_{R_{2}}$ the standard deviations of relative risks of equine and human WNv; while dot denotes the inner product of the two vectors $R_{1}$ [ ] and $R_{2}$ [] (Lawson, 2009). Humphreys County and Tunica County were excluded from all analyses owing to missing data in the 2002 Mississippi equine census; thus $N=80$. The expected counts of human WNv cases from Wang et al. (2010) were used in this study. The MCAR models were implemented with the WinBUGS 1.4.3 software (Spiegelhalter et al., 2002b). Two MCMC chains for spatial correlation $C R$ were initialized with different starting values, and run for 30,000 iterations with the first 20,000 iterations as the burn-in period. The Books-Gelman-Rubin method was used to assess the convergence of the two chains (Spiegelhalter et al., 2002b).

\section{Results}

A total of 256 cases of equine $\mathrm{WNv}$ infections were reported in Mississippi in 2002. Thirty-two counties had zero cases, and 40 counties had <10 cases in 2002 (Figure 1). The model without the $\mathrm{CH}$ spa-

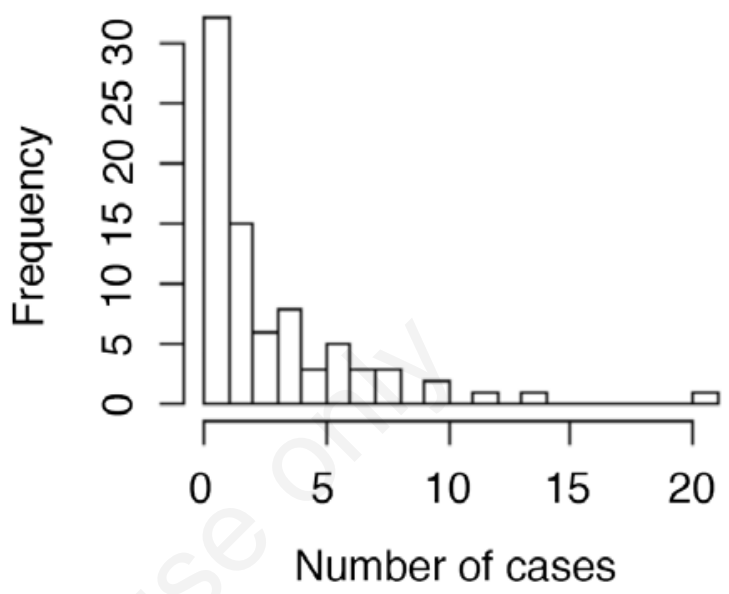

Figure 1. Histogram of the number of the cases of equine West Nile virus infections of Mississippi at the county level in 2002.

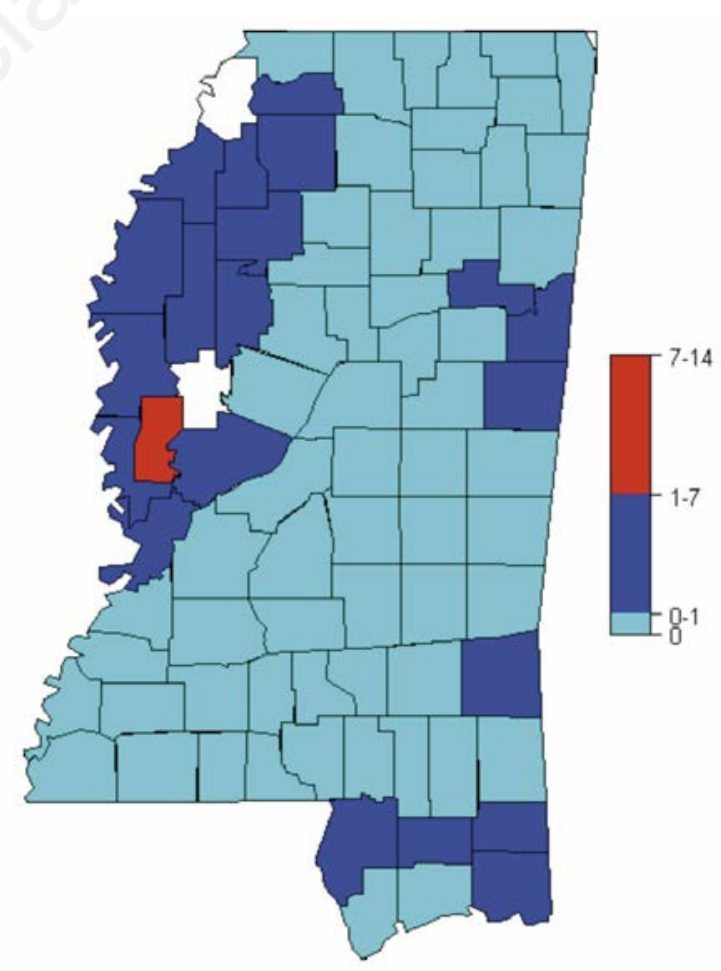

Figure 2. Relative risk of equine West Nile virus (WNv) of 2002 in Mississippi, USA, estimated by Bayesian hierarchical models. Counties with values of relative risk exceeding 1 had WNv risk greater than expected. The breaks (0-1, 1-7, and 7-14) of the legend are the ranges of the relative risk. Humphreys County and Tunica County (marked with white colour) were excluded from the analysis owing to missing data in the 2002 horse census. 
tial effect (i.e., CAR distribution) had the largest DIC value and lowest DIC weight, indicating that the model without the spatial component received the least support from data among the candidate models (Table 1). The DIC values of models with $\mathrm{CH}$ and $\mathrm{UH}$ indicated the necessity of CAR models for the relative risk of equine WNv infection (Table 1). In a preliminary analysis, a model including annual precipitation of both the previous and current year did not explain more variability than did a model that only included precipitation of the previous year. Thus, precipitation of the current year was not included in the subsequent analysis. Two competing model had $\triangle \mathrm{DIC}<2.0$ and the DIC weight of 0.41 ; one included annual precipitation of the previous year and the other annual precipitation of the previous year plus land cover diversity index as covariates (Table 1). Identical DIC weight suggested that the two models received equal support from data. The $95 \%$ credible interval (CI) of the coefficient of annual precipitation and land cover diversity index were below zero in the two-covariate competing model (Table 1). Exclusion of zero from the $95 \%$ CIs indicated significant, negative effects of increased precipitation and land cover diversity with regard to equine WNv infection risks. Estimated relative risks of equine WNv varied over Mississippi in 2002 with the Mississippi Alluvial Valley north and central-west of Mississippi having greater risk than that of the other regions (Figure 2). Twenty-one counties had the relative risk of greater than 1.0 (Figure 2). Additionally, the relative risks of equine and human $\mathrm{WNv}$ were positively correlated, with the mean correlation (CR) of 0.22 (95\% CI: $0.13-0.46)$.

Additionally, this study demonstrated that the application of Bayesian hierarchical models implemented with recently developed integrated nested Laplace approximation (INLA) methods at countylevel mapping of equine WNv infection risks (Rue et al., 2009) is useful. The INLA methods avoid the convergence problem of popular Markov chain Monte Carlo (MCMC) approaches to Bayesian inferences (Rue et al., 2009).

\section{Discussion}

Weather and landscape structure affected equine WNv infections in Mississippi in 2002. Precipitation exhibited the delayed negative effects on equine WNv infection rates. Increased landscape diversity reduced equine WNv infections (Table 1). However, DeGroote et al. (2014) found that high winter and spring precipitation followed by dry summer enhanced human WNv infection risks in the south-eastern USA in 2012. Dry weather conditions probably reduced mosquito predators (e.g., fish and amphibians) and competitors (Chase and Knight, 2003). Consequently, mosquitoes may thrive due to reduced predation and competition during the period between local extinction and recolonisation of predators and competitors in temporary standing- water habitats (Chase and Knight, 2003). Alternatively, $C$. pipiens and C. quinquefasciatus are common mosquitos in Mississippi and are also important WNv vectors (Kothera et al., 2009; Farajollahi et al., 2011; Andreadis, 2012). C. pipiens breeds better in drier weather than under wetter conditions because rain flushes $C$. pipiens larvae out of manmade containers, tires or tire ruts, thus reducing $C$. pipiens abundance (Koenraadt and Harrington, 2008). Dry weather of the previous year may increase $C$. pipiens and $C$. quinquefasciatus abundance the following year (Landesman et al., 2007; Van Dam and Walton, 2008). Studies found that equine WNv infections in $C$. pipiens were inversely related to the precipitation of the previous year (Landesman et al., 2007; Ruiz et al., 2010). Weather-induced increases in mosquito abundance and $\mathrm{WNv}$ infections in turn increased the transmission of WNv to horses.

Land use and land cover are important factors influencing the spatial heterogeneity of WNv infection risk (Brown et al., 2008a, 2008b; Pradier et al., 2008). Increases in land cover diversity enhance the species diversity of birds in the State of Louisiana (Ezenwa et al., 2007). Equine WNv infection risk decreased with increased land cover diversity index, probably because of the diluting effects of high avian species diversity in areas with diverse land covers. Therefore, it is possible to use habitat management practices aimed to increase land cover diversity or biodiversity of ecosystems to reduce the infection risks of WNv or other vector-borne diseases in the long run (LoGiudice et al., 2003; Keesing et al., 2010; Roche et al., 2012).

Although the relative risks of human and equine WNv infections were both related to annual precipitation of the previous year (this study; Wang et al., 2010), the correlation of relative WNv risks between humans and horses was low $(=0.22)$ in Mississippi in 2002. The value of the precipitation coefficient was -0.005 in the human WNv model (Wang et al., 2010), but it was -0.012 in the equine WNv model (Table 1). Dry weather conditions may exert stronger effects with regard to equine WNv risk than to human WNv risk in agricultural areas. The relative risk of human WNv infection in central Mississippi was highest within Mississippi in 2002 (Wang et al., 2010), whereas the relative risk of equine WNv infection in the Mississippi Alluvial Valley was highest in the same year (Figure 2; Wang et al. 2010). The difference of WNv infection between humans and horses in the hotspots was probably because the central part of Mississippi includes the Jackson and Meridian areas with the greatest human population density of the state, whereas the Mississippi Alluvial Valley is a rural area dominated by row crop fields. Increased percentage of urban land increases human WNV risk in the north-eastern US (Brown et al., 2008b). Although human cases are concentrated in cities and surrounding areas, people may be infected in places other than the place of residence. Additionally, humans in urban areas and horses in rural areas may have different mosquito species as the main bridge vectors of WNv transmission from infected birds (Ward and Scheurmann, 2008). Under dry weather conditions, temporal standing water in tire tracks

Table 1. Bayesian hierarchical models for the effects of annual precipitation of the previous year and land cover diversity on the relative risk of equine West Nile virus in Mississippi, USA, in 2002.

\begin{tabular}{lcccc} 
Model & DIC & $\Delta \mathrm{DIC}$ & Weight & Mean and 95\% CI of coefficient \\
$c+\mathrm{UH}$ & 333.83 & 7.12 & 0.01 & $\mathrm{NA}$ \\
$\mathrm{c}+\mathrm{UH}+\mathrm{CH}$ & 328.47 & 1.76 & 0.17 & $\mathrm{NA}$ \\
\hline$c+$ prec $+\mathrm{UH}+\mathrm{CH}$ & 326.71 & 0 & 0.41 & $-0.012(-0.025,0.001)$ \\
$c+$ prec + div $+\mathrm{UH}+\mathrm{CH}$ & 326.72 & 0.01 & 0.41 & prec: $-0.012(-0.024,-0.001) ;$ div: $-1.81(-2.323,-1.284)$ \\
\hline
\end{tabular}

DIC, deviance information criterion; $\triangle \mathrm{DIC}$, difference between the DIC of a candidate model and the lowest DIC; weight, equivalent to Akaike information criterion; CI, confidence interval; $c$, constant; UH, uncorrelated heterogeneity; NA, not available; $\mathrm{CH}$, correlated heterogeneity modelled by conditional autocorrelative distribution; prec, annual precipitation of 2001 ; div, diversity index of land cover types. 
and irrigation water in the crop fields of the Mississippi Alluvial Valley probably provide suitable breeding habitat for the mosquitoes responsible for WNv transmission to horses (Mongoh et al., 2007). Therefore, horses in the Mississippi Alluvial Valley are more vulnerable to WNv than those in other areas of Mississippi.

\section{Conclusions}

There is growing evidence that climate and dry weather may modulate the risk of human and horse WNv outbreaks spatially and temporally (This study; Landesman et al., 2007; Wang et al., 2010; AharonsonRaz et al., 2014). Wimberly et al. (2008) found a quadratic relationship between the rate of human WNv diseases in 2003 and May-July precipitation of 2002 in the Great Plains in USA, with the 2003 rate of human WNv diseases decreasing when total May-July precipitation reached beyond $200 \mathrm{~mm}$. In addition, the land use and land cover of a region are unlikely to change at a large spatial scale in the short term (e.g., 1-2 years) unless catastrophic disasters occur. Therefore, annual precipitation of the previous year (with either positive or negative effects) may be used as a predictor for the early warning of human and equine WNv at the regional level. This study used the 2002 data on equine WNv outbreaks when the virus initially invaded and swept through Mississippi to reveal ecological effects of dry weather conditions on the WNv outbreaks. Thus, the results of this study may have implications limited only to equine WNv outbreaks. Other environmental factors (e.g., temperature, the composition of WNv vector communities, topography and farming practices), which were not included in this study, may also influence equine WNv risks in Mississippi. In addition, the vaccination status of horses may affect the WNv infection risk of equids. Future studies should address the region-specific effects of dry weather conditions on the $\mathrm{WNv}$ prevalence of mosquitoes and interactions of precipitation and land cover on the WNv infection risks to better understand mechanisms underlying climate effects on the transmission of WNv to mammals.

\section{References}

Aharonson-Raz K, Lichter-Peled A, Tal S, Gelman B, Cohen D, Klement E, Steinman A, 2014. Spatial and temporal distribution of West Nile virus in horses in Israel (1997-2013). From endemic to epidemics. PLoS One 9:e113149.

Andreadis TG, 2012. The contribution of Culex pipiens complex mosquitoes to transmission and persistence of west nile virus in North America. J Am Mosquito Contr 28:137-51.

Blangiardo M, Cameletti M, Baio G, Rue H, 2013. Spatial and spatiotemporal models with R-INLA. Spat Spatio-temporal Epidemiol 4:33-49.

Brown H, Duik-Wasser M, Andreadis T, Fish D, 2008a. Remotely-sensed vegetation indices identify mosquito clusters of West Nile virus vectors in an urban landscape in the northeastern United States. Vector-Borne Zoonot 8:197-206.

Brown HE, Childs JE, Diuk-Wasser MA, Fish D, 2008b. Ecological factors associated with West Nile virus transmission, northeastern United States. Dis 14:1539-45.

Burnham, KP, Anderson, DR, 2002. Model selection and inference: a practical information-theoretic approach. 2nd ed. Springer-Verlag, New York, NY, USA.

Chase JM, Knight TM, 2003. Drought-induced mosquito outbreaks in wetlands. Ecol Lett 6:1017-24.

Cooke WH, Grala K, Wallis RC, 2006. Avian GIS models signal human risk for West Nile virus in Mississippi. Int J Health Geogr 5:36.

DeGroote JP, Sugumaran R, Ecker M, 2014. Landscape, demographic and climatic associations with human West Nile virus occurrence regionally in 2012 in the United States of America. Geospat Health 9:153-68.

Epstein PR, Defilippo C, 2001. West Nile virus and drought. Glob Change Hum Health 2:105-7.

Ezenwa V0, Milheim LE, Coffey MF, Godsey MS, King RJ, Guptill SC, 2007. Land cover variation and West Nile virus prevalence: patterns, processes, and implications for disease control. Vector-Borne Zoonot 7:173-80.

Farajollahi A, Fonseca DM, Kramer LD, Kilpatrick AM, 2011. "Bird biting" mosquitoes and human disease: a review of the role of Culex pipiens complex mosquitoes in epidemiology. Infect Genet Evol 11:1577-85.

Harrigan RJ, Thomassen HA, Buermann W, Smith TB, 2014. A continental risk assessment of West Nile virus under climate change. Glob Change Biol 20:2417-25.

Hayes EB, Komar N, Nasci RS, Montgomery SP, 0'Leary DR, Campbell GL, 2005. Epidemiology and transmission dynamics of West Nile Virus disease. Emerg Infect Dis 11:1167-73.

Homer C, Dewitz J, Fry J, Coan M, Hossain N, Larson C, Herold N, McKerrow A, VanDriel JN, Wickham J, 2007. Completion of the 2001 National Land Cover Database for the counterminous United States. Photogramm Eng Rem S 73:337-41.

Keesing F, Belden LK, Daszak P, Dobson A, Harvell CD, Holt RD, Hudson P, Jolles A, Jones KE, Mitchell CE, Myers SS, Bogich T, Ostfeld RS, 2010. Impacts of biodiversity on the emergence and transmission of infectious diseases. Nature 468:647-52.

Koenraadt C, Harrington L, 2008. Flushing effect of rain on containerinhabiting mosquitoes Aedes aegypti and Culex pipiens (Diptera: Culicidae). J Med Entomol 45:28-35.

Kothera L, Zimmerman EM, Richards CM, Savage HM, 2009. Microsatellite characterization of subspecies and their hybrids in Culex pipiens complex (Diptera: Culicidae) mosquitoes along a north-south transect in the central United States. J Med Entomol 46:236-48.

LaDeau SL, Marra PP, Kilpatrick AM, Calder CA, 2008. West Nile Virus revisited: consequences for North American ecology. Bioscience 58:937-46.

Landesman WJ, Allan BF, Langerhans RB, Knight TM, Chase JM, 2007. Inter-annual associations between precipitation and human incidence of West Nile virus in the United States. Vector-Borne Zoonot 7:337-43.

Lawson AB, 2009. Bayesian disease mapping: hierarchical modeling in spatial epidemiology. CRC Press, Boca Raton, FL, USA.

Lawson AB, Browne WJ, Vidal-Rodiero CL, 2003. Disease mapping with WinBUGS and MLwiN. Wiley, New York, NY, USA.

LoGiudice K, Ostfeld RS, Schmidt KA, Keesing F, 2003. The ecology of infectious disease: effects of host diversity and community composition on lyme disease risk. PNAS 100:567-71.

MacArthur RH, 1964. Environmental factors affecting bird species diversity. Am Nat 98:387-97.

Mongoh MN, Khaitsa ML, Dyer NW, 2007. Environmental and ecological determinants of West Nile virus occurrence in horses in North Dakota, 2002. Epidemiol Infect 135:57-66. 
Nash D, Mostashari F, Fine A, Miller J, O'Leary D, Murray K, Huang A, Rosenberg A, Greenberg A, Sherman M, Wong S, Layton M, Campbell GL, Roehrig JT, Gubler DJ, Shieh WJ, Zaki S, Smith P, 2001. The outbreak of West Nile virus infection in the New York City area in 1999. New Engl J Med 344:1807-14.

0'Leary DR, Marfin AA, Montgomery SP, Kipp AM, Lehman JA, Biggerstaff BJ, Elko VL, Collins PD, Jones JE, Campbell GL, 2004. The epidemic of West Nile virus in the United States, 2002. VectorBorne Zoonot 4:61-70.

Ostfeld RS, Keesing F, 2000. The function of biodiversity in the ecology of vector-borne zoonotic diseases. Can J Zool 78:2061-78.

Pradier S, Leblond A, Durand B, 2008. Land cover, landscape structure, and West Nile virus circulation in southern France. Vector-Borne Zoonot 8:253-63.

Ribeiro J, Diggle PJ, 2001. geoR: a package for geostatistical analysis. Available from: http://www.leg.ufpr.br/geoR/

Rios LMV, Sheu JJ, Day JF, Maruniak JE, Seino K, Zaretsky H, Long MT, 2009. Environmental risk factors associated with West Nile virus clinical disease in Florida horses. Med Vet Entomol 23:357-66.

Roche B, Dobson AP, Guégan J, Rohani P, 2012. Linking community and disease ecology: the impact of biodiversity on pathogen transmission. Philos T R Soc B 367:2807-13.

Rue H, Martino S, Chopin N, 2009. Approximate Bayesian inference for latent Gaussian models by using integrated nested Laplace approximations. J R Stat Soc B Met 71:319-92.

Ruiz M0, Chaves LF, Hamer GL, Sun T, Brown WM, Walker ED, Haramis L, Goldberg TL, Kitron UD, 2010. Local impact of temperature and precipitation on West Nile virus infection in Culex species mosquitoes in northeast Illinois, USA. Parasite Vector 3:19.

Schuler LA, Khaitsa ML, Dyer NW, Stoltenow CL, 2004. Evaluation of an outbreak of West Nile virus infection in horses: 569 cases (2002). J Am Vet Med Assoc 225:1084-9.

Spiegelhalter DJ, Best NG, Carlin BR, van der Linde A, 2002a. Bayesian measures of model complexity and fit. J R Stat Soc B Met 64:583616.

Spiegelhalter DJ, Thomas A, Best NG, Lunn D, 2002b. WinBUGS user manual. Version 1.4. MRC Biostatistics Unit, Cambridge, UK.

Van Dam A, Walton W, 2008. The effect of predatory fish exudates on the ovipostional behaviour of three mosquito species: Culex quinquefasciatus, Aedes aegypti and Culex tarsalis. Med Vet Entomol 22:399-404.

Walsh MG, 2012. The role of hydrogeography and climate in the landscape epidemiology of West Nile virus in New York State from 2000 to 2010. PloS One 7:e30620.

Wang GM, Minnis RB, Belant JL, Wax C, 2010. Dry weather induces outbreaks of human West Nile virus infections. BMC Infect Dis 10:38.

Ward MP, Scheurmann JA, 2008. The relationship between equine and human West Nile virus disease occurrence. Vet Microbiol 129:37883.

Wimberly MC, Hildreth MB, Boyte SP, Lindquist E, Kightlinger L, 2008. Ecological niche of the 2003 West Nile virus epidemic in the Northern Great Plains of the United States. PloS One 3:e3744. 